\title{
Staff training: a key factor in reducing intravascular catheter sepsis
}

\author{
J W L Puntis, C E Holden, S Smallman, Y Finkel, R H George, I W Booth
}

\begin{abstract}
A children's hospital nutritional care team prospectively monitored the frequency of sepsis in central venous catheters used for administering parenteral nutrition. During an initial study period of 12 months, $26 / 58(45 \%)$ of catheters were removed because of proved sepsis. The possible causes of this alarmingly high rate were examined, with catheter care techniques on the wards coming under particular scrutiny. As a result protocols were modified and an intensive staff training programme implemented throughout the hospital, led by the nutritional care sister. Subsequently, the catheter sepsis rate was significantly reduced with only $9 / 107(8 \%)$ of consecutive catheters becoming infected. These findings emphasise the key role that education of staff plays in controlling central venous catheter sepsis and the importance and cost effectiveness of special nursing staff in implementing such measures.
\end{abstract}

Central venous catheterisation provides reliable venous access during parenteral nutrition and permits the administration of hypertonic solutions. Catheter related sepsis remains an important unsolved problem ${ }^{12}$ and results in significant morbidity and mortality. Infection control measures should include periodic prospective review of sepsis rates. In our hospital all parenteral nutrition is prescribed by a member of the nutritional care team ${ }^{3}$; this team also monitors complications related to nutritional support. Over a 12 month period (study 1) it became apparent that there had been a considerable increase in the rate of catheter sepsis above the $15 \%$ previously determined. ${ }^{4} \mathrm{~A}$ number of different strategies for preventing sepsis have been proposed, 5 some of which have not been proved effective in practice. ${ }^{67}$ The importance of impeccable catheter care, although difficult to define, is generally regarded as being crucial. We therefore determined to focus attention on existing central venous catheter care protocols and examine the way they were applied on the wards. This study describes our investigation of the epidemic, the ways in which we sought to improve matters, and the results of a planned education programme (study 2).

\section{Subjects and methods}

STUDY 1

Forty consecutive patients referred to the nutritional care team for parenteral feeding were included. Two thirds had undergone gastrointestinal surgery, most in the neonatal period.
Cardiac surgery accounted for $20 \%$ and cardiac, oncology, and gastroenterology patients made up the rest. The median (range) age at starting parenteral feeding was 15 days ( 2 days -9 years).

A total of 58 central venous catheters were studied, comprising equal numbers of percutaneous Silastic ${ }^{8}$ and Broviac catheters. Percutaneous catheters were inserted on the ward and a dry dressing applied to the skin entry site before taping in place with adhesive plaster. The skin entry site was not routinely inspected unless there were clinical suspicions of catheter sepsis, and dressings were not routinely changed unless soiled or loose. Broviac catheters were inserted under general anaesthesia in an operating theatre, usually by cutdown onto an internal jugular vein. A clear adhesive dressing (Opsite, Smith and Nephew) was then applied. All catheters were sited with their tip just within the right atrium.

Catheters were used exclusively for parenteral nutrition and not for administration of drugs or blood sampling. Parenteral feed source containers were changed every 24 hours and a 0.2 micron filter used in line with the amino acid/ dextrose preparation. In the event of the catheter hub being disconnected (occlusion requiring flushing, or filter breakage), feed solutions were replaced with $10 \%$ dextrose until the next day's feed prescription became available.

A diagnosis of probable catheter sepsis was made in a feverish or unwell patient without evidence of focal infection such as otitis or pneumonia, and when urine culture was negative. Primary catheter sepsis was then considered to be proved when peripheral blood cultures were positive for bacterial pathogens. Treatment involved catheter removal and intravenous antibiotics, usually vancomycin and gentamicin, until sensitivities of organisms were available. Non-quantitative bacteriological techniques were used for blood cultures. Samples were placed in brain heart infusion broth (Oxoid UK Ltd) for aerobic culture, and thyoglycollate medium USP (Oxoid UK Ltd) for anaerobic culture. Broth bottles were incubated at $37^{\circ} \mathrm{C}$ and examined each day for evidence of growth. Positive cultures were subcultured to blood agar and examined by Gram film. All bottles were routinely subcultured at 48 hours and again after seven days' incubation. Fungal organisms were routinely sought.

Catheter tips were rolled across the surface of $5 \%$ blood agar plates, using the technique of Maki $e t a l,{ }^{9}$ and were then incubated aerobically at $37^{\circ} \mathrm{C}$. Using this method colony counts greater than 15 were considered additional evidence for catheter sepsis. The final diagnosis 
was based on positive blood cultures and clinical features, including symptomatic improvement after catheter removal. In four cases of catheter sepsis occurring in patients on long term parenteral feeding, antibiotic treatment was given with the catheter left in situ. In two instances blood cultures remained positive at $\mathbf{4 8}$ hours and the catheter was then removed, and on two occasions after 48 hours of treatment, blood cultures remained negative and antibiotics were successfully discontinued after two weeks with the catheter left in situ.

\section{Education programme}

Staff communication-After the analysis of the results in study 1 , meetings were held between the nutritional care team and consultant microbiologist and staff from medical and surgical units. Routines for changing parenteral feed infusions and dealing with blocked lines were scrutinised by the microbiologist as they were being performed on the ward.

Hand washing-Forty per cent of medical and nursing staff were observed to follow an incomplete hand washing technique and efforts were directed at teaching correct hand washing procedure. ${ }^{10}$

Bag changes-After their usual practice of hand washing for two minutes in antiseptic soap (Hibiscrub), nurses were asked to start wearing sterile gloves. In addition to immersing the catheter hub in $70 \%$ isopropyl alcohol before disconnection, it was decided that the tubing up to $20 \mathrm{~cm}$ on either side of the catheter hub should also be cleaned with a gauze soaked in alcohol and then allowed to dry.

New guidelines for catheter care were drawn up, ${ }^{11}$ emphasising the importance of aseptic technique, and the importance of minimising hub disconnections and line violations. It was agreed that two nurses rather than one should always be involved with changing parenteral nutrition feed bags so that one could handle only sterile equipment and the other 'dirty' equipment. This practice had lapsed under pressure of work on the ward where most parenterally fed patients were cared for, and only one nurse had been changing feeding bags.

Training-Given the high turnover of medical and nursing staff it was apparent that training programmes should recur at regular intervals so that new medical and nursing staff could be taught correct procedures. The need for junior medical staff to seek guidance from senior ward nurses on line care, for example when flushing a blocked central venous catheter, was emphasised and accepted.

A further series of meetings was arranged between medical and surgical teams and nutritional care sister to emphasise the importance of central venous catheter care, and arrangements made for this topic to be covered at each induction day for new medical and surgical staff. Practical demonstrations of line care and bag changing were organised for all nursing staff. Attendance was compulsory, and training carried out over a three month period. The nutri- tional care sister and clinical support nursing officer also arranged practical demonstrations of line care on all those wards regularly dealing with central lines. A video film showing bag changing and line flushing was also made by the department of medical illustration and the nutritional care sister and used in subsequent teaching sessions.

\section{STUDY 2}

After the intensive education programme, 92 patients were studied with a median age (range) at the start of parenteral feeding of 13 days ( 1 day-15 years). Seventy five $(81 \%)$ patients were under 1 year of age and most $(50,54 \%)$ were recovering from gastrointestinal surgery; $24(26 \%)$ had had cardiac surgery and the remaining patients had various different indications for nutritional support. In all, 107 central lines were inserted, and these comprised 35 triple lumen and 12 double lumen Vygon catheters, 34 percutaneous Silastic, and 26 Broviac catheters. Vygon catheters were inserted percutaneously either in theatre or on the intensive care unit and one channel devoted exclusively to administration of parenteral feeding solutions. Otherwise catheter insertion, dressing, and parenteral feeding protocols together with criteria for diagnosing sepsis were the same as in study 1 .

\section{STATISTICAL METHODS}

Comparisons of the ages of patients at the start of parenteral nutrition and of catheter life was made using the Mann-Whitney U test. The incidence of catheter sepsis in each study was compared using the $\chi^{2}$ test.

\section{Results}

STUDY 1 (BEFORE EDUCATION PROGRAMME)

The median (range) catheter life was 15 (3-66) days, giving a total of 963 catheter days in all. Thirteen of each type of catheter $(26 / 58,45 \%)$ became primarily infected, representing one episode of sepsis every 42 catheter days. Infections occurred sporadically after line insertion and no significant difference was found comparing the duration of catheter life in infected and non-infected catheters $(p=0.64)$. In 23 cases, coagulase negative staphylococci were isolated from blood and/or line, with Staphylococcus aureus being found in two cases and Escherichia coli in one.

STUDY 2 (AFTER EDUCATION PROGRAMME)

The median (range) catheter life was 10 (1-84) days giving a total of 1684 catheter days in all. Now, only $9 / 107$ ( $8 \%$ ) of catheters became primarily infected (five percutaneous Silastic, three Vygon, one Broviac), a significantly lower number than before the education programme $(p<0.001)$ representing one episode of sepsis/ 189 days of catheter use. Again, infections occurred sporadically after catheter insertion and the life of infected catheters was not significantly greater than that of non-infected catheters $(p=0 \cdot 17) . E$ coli was isolated from four cases of catheter sepsis, coagulase negative staphylococci from three, and Klebsiella aerogenes and Streptococcus viridans from the remaining two. 
There was no significant difference found when comparing the age of patients at the start of parenteral feeding in each group $(p=0.24)$ but catheter life was significantly shorter in the patients in study $2(\mathrm{p}=0.01)$.

\section{Discussion}

The first part of this study showed that catheter sepsis rates among parenterally fed patients had increased from $15 \%$ to $45 \%$, after a period when catheter infection had not been closely monitored. An intensive programme of staff education was then initiated, which emphasised the importance of scrupulous aseptic technique and led to several changes in catheter care protocols. We cannot, of course, be sure about the precise mechanisms by which our education programme brought about such a dramatic reduction in catheter sepsis rates from $45 \%$ to $8 \%$ (see table). Perhaps the most important of these was the requirement that two people should be involved with each central venous catheter disconnection and feed bag change. Although this had previously been our recommendation, the practice had lapsed during a period when increasing numbers of patients were cared for by fewer nursing staff.

The reduction in catheter sepsis after implementation of the education programme could not be accounted for by other factors. The age of patients and clinical indications for feeding were much the same in each part of the study. Although the length of time for which catheters were in situ was significantly greater in study 1 , in neither study was there a simple association between days in situ and risk of infection. Sepsis occurred sporadically after insertion, and no significant difference was found comparing catheter life in non-infected and infected catheters. Although Vygon percutaneous catheters were used during study 2 , the reduction in catheter sepsis remains unchanged even if these are excluded from the analysis.

In controlling complications of parenteral nutrition, particularly catheter related sepsis, the key role of specially trained nursing staff, ${ }^{7}{ }^{12}$ often in the context of a nutritional care team, ${ }^{13}$ has gained widespread recognition, together with the appreciation that they are cost effective. Based on the assumption that patients who develop catheter sepsis probably stay on average a week longer in hospital than they would otherwise have done, the cost saving brought about by our intervention study through a reduction in infection over the next year would have been approximately $£ 80000$ ( $\$ 870.00$ per parenterally fed patient). This takes into account the cost of antibiotic treatment and mean cost of one week's inpatient care.

Factors which may help reduce central venous catheter sepsis

\begin{tabular}{|c|c|}
\hline Prospective audit & Warns of adverse trends \\
\hline Catheter use & $\begin{array}{l}\text { Rases staft awareness } \\
\text { Dedicated to parenteral feeding } \\
\text { Minimal line violation }\end{array}$ \\
\hline Bag changes & $\begin{array}{l}\text { Two nurse system } \\
\text { Sterile gloves }\end{array}$ \\
\hline Training & $\begin{array}{l}\text { Recurrent staff training programme } \\
\text { Ward demonstrations } \\
\text { Video film } \\
\text { Central role of nutritional care nurse }\end{array}$ \\
\hline
\end{tabular}

Infecting organisms come primarily from the catheter hub, ${ }^{14-16}$ and studies which have concentrated on minimising the risk of bacterial hub contamination have shown significant reductions in sepsis rate. ${ }^{17}$ Catheter infection by organisms gaining access to the bloodstream from the skin entry site is relatively uncommon; this explains why attempts to reduce sepsis rates by topical application of antibiotic and antiseptic agents, ${ }^{6}$ or by bringing the catheter out through a skin tunnel, ${ }^{18}$ have not been shown to be effective.

Prevention of central venous catheter sepsis is particularly difficult in infants, who often have skin contaminated with faeces, and who tend to play with or chew on catheters and connections. There is a natural reluctance on the part of many units to publish figures showing a high catheter sepsis rate, although some have reported experiences similar to our own. ${ }^{19} 20$ We suspect that even collection of such data is not routine in many centres and yet our study, the first of its kind in children, emphasises how infection rates can suddenly increase. Careful prospective monitoring of catheter sepsis is essential if adverse trends are to be reliably identified at an early stage and then reversed.

1 Maki DG. Sepsis associated with infusion therapy. In: Karran S, ed. Controversies in surgical sepsis. Eastbourne: Karran S, ed. Controversies in surge

2 Bozzetti F. Central venous catheter sepsis. Surg Gynecol Obstet 1985;161:293-301.

3 Puntis JWL, Booth IW. The place of a nutritional care team in paediatric practice. Intensive Therapy and Clinical Monitoring 1990;11:132-6.

4 Puntis JWL. Percutaneous insertion of Silastic central venous feeding catheters. Intensive Therapy and Clinical Monitoring 1987;8:7-10.

5 Goldman DA, Maki DG. Infection control in total parenteral nutrition. FAMA 1973;223:1361-4.

6 Jarrard MM, Freeman JB. The effects of antibiotic ointments and antiseptics on the skin flora beneath subclavian catheter dressings during intravenous hyperalimentation. ₹ Surg Res 1977;22:521-6.

7 Keohane PP, Attrill H, Northover J, et al. Effect of catheter tunnelling and nutrition nurse on catheter sepsis during tunnelling and nutrition nurse on catheter

8 Shaw JCL. Parenteral nutrition in the management of sick, low birthweight infants. Pediatr Clin North Am 1973;20: 333-58.

9 Maki DG, Weise CE, Sarafin HW. A semiquantitative culture method for identifying intravenous-catheter-related infection. N Engl f Med 1977;296:1305-9.

10 Taylor LJ. An evaluation of hand washing techniques-1. Nursing Times January 1978:54-5.

11 Ball PA, Booth IW, Puntis JWL. Paediatric parenteral nutrition. Bourne End: KabiVitrum, 1989:43-7.

12 Haddock G, Barr J, Burns HJG, Garden OJ. Reduction of central venous catheter complications. British foumal of Parenteral Therapy 1985;6:124-7.

13 Hamaoui E. Assessing the nutrition support team. Fournal of Parenteral and Enteral Nutrition 1987;11:412-21.

14 Sitges-Serra A, Puig P, Linares J, et al. Hub colonisation as the initial step in an outbreak of catheter-related sepsis due to coagulase negative staphylococci during parenteral to coagulase negative staphylococci during parenteral 8:668-72.

15 Sitges-Serra A, Linares J. Tunnels do not protect against catheter related sepsis. Lancet 1984; :459-60.

16 Linares J, Sitges-Serra A, Garau J, Perez JL, Martin R. Pathogenesis of catheter sepsis: a prospective study with Pathogenesis of catheter sepsis: a prospective study with
quantitative and semiquantitative cultures of catheter hub quantitative and semiquantitative cultures of ca

17 Stotter AT, Ward H, Waterfield AH, Hilton J, Sim AJW. Junctional care: the key to prevention of catheter sepsis in Junctional care: the key to prevention of catheter sepsis in intravenous feeding. Jou

18 Garden OJ, Sim AJW. A comparison of tunnelled and nontunnelled subclavian vein catheters: a prospective study of complications during parenteral feeding. Clinical Nutrition 1983;2:51-4.

19 Kiely E. One hundred consecutive central venous catheters in children. Z Kinderchir 1984;39:332-6.

20 Glass EJ, Hume R, Lang MA, Forfar JO. Parenteral nutrition compared with transpyloric feeding. Arch Dis Child 1984;59:131-5. 